\title{
Typing Asymmetric Client-Server Interaction ${ }^{\star}$
}

\author{
Franco Barbanera ${ }^{1}$, Sara Capecchi $^{2}$, and Ugo de'Liguoro ${ }^{2}$ \\ 1 Dipartimento di Matematica e Informatica, Università di Catania \\ ${ }_{2}$ Dipartimento di Informatica, Università di Torino \\ ${ }^{1}$ barba@dmi . unict.it, ${ }^{2}$ \{capecchi, deligu\}@di.unito.it
}

\begin{abstract}
We investigate client-server interaction where duties and rights of the parties are asymmetric, in the sense that the client is allowed to abort any session before the server has completed, but not vice versa. This implies that the client can interact with any server offering at least what she is looking for, but possibly more.

We formalize such asymmetry in the setting of session types via a form of subtyping in depth, which we call prefix relation. This is apparently conflicting with the rigid duality imposed by session types; nonetheless the resulting system retains all basic correctness properties.

Moreover, the system we propose highlights interesting aspects concerning the flow of communication inside a session. In particular it reveals that usual subtyping theories cannot be extended by means of prefix, which turns out to be a different concept.
\end{abstract}

Key words: Process calculi, Type Systems, Session Types, Client/Server Interaction Protocols, Subtyping, Contro/Covariance

\section{Introduction}

Client/server is the relationship between two software applications in which one of them, the client, addresses its request of a service to the other one, the server, which is expected to fulfill the request. It is an intrinsically asymmetric relationship, not just because there is one interacting end that provides a service and the other that makes use of it. There are also differences in the rights and duties of the parties: it is indeed unreasonable to prohibit to the client to abort the connection at any time, while it would be unfair to admit such a behavior on the server side.

In [4] a theory of contracts is proposed in order to formalize the search for a service on the web and to discipline the client/server relationship. A contract is an abstract specification of the service. A client will comply with a service if it will successfully terminate any interaction with the service, which however might provide more. Since (the dual of) a contract is able to specify the interaction protocol also on the client side, the compliance of the client with the server can be checked by formally proving that the dual of the client contract is (with some

\footnotetext{
* This work has been partially supported by the MIUR project EOS-DUE.
} 
simplification) the initial part of the server contract. The same asymmetry is at the heart of the subcontract relationship studied in [4].

Following the suggestion in [15], we consider types as a natural candidate to formalize contracts, and more precisely the session types introduced in [8], a type system for a dialect of the $\pi$-calculus adding primitives to handle sessions. A session is an abstraction of a sequence of communications through a private channel between two parties. It is created by connecting over a session channel (often called live channel) in such a way that both privacy and duality are guaranteed. The type system is then used to abstract a discipline of the interaction into a session type and to ensure safe handshaking-communications.

The idea is that of changing the interpretation of the typing $x: S$, where $S$ is a session type, according to the cases of $x$ being the server or the client end in a session. In the server case $S$ represents its duties, namely the commitment to an interaction which is at least of the shape (and the length) represented by $S$. If $x$ is instead the client end, then $S$ represents the client's rights, telling that it is entitled to ask at most an interaction of that shape.

According to the system in [8] sessions have to be symmetric. A simple example of symmetric interaction is between the following sketchy calculator server and one of its possible clients:

$$
\begin{aligned}
\text { CalcServer }_{1}={ }_{\text {def }} \text { accept } a(x) \cdot x \triangleright\{ & \text { add }: x ?(n) \cdot x ?(m) \cdot x ![n+m], \\
& \ldots \\
& \operatorname{div}: x ?(n) \cdot x ?(m) \cdot x ![n \operatorname{div} m]\}
\end{aligned}
$$

CalcClient $_{1}=_{\text {def }}$ request $a(x) . x \triangleleft \operatorname{div} . x ![21] . x ![5] . x ?(n)$

The type of the name $x$ along which a client might interact with CalcServer $_{1}$ is

$$
S_{\text {server1 }}=\&\langle\text { add : ?(int) ?(int)![int]end, .., div : ?(int) ?(int)![int]end }\rangle,
$$

while the type of the $x$ on the side of CalcClient $_{1}$ is exactly its dual:

$$
S_{\text {client } 1}=\overline{S_{\text {server } 1}}=\oplus\langle\text { add :![int] ![int] ?(int)end, ..., div : ! [int] ![int] ?(int)end }\rangle
$$

However, the service request of $\mathrm{CalcClient}_{1}$ would be satisfied also by the server

$$
\begin{aligned}
\text { CalcServer }_{2}= & \text { def accept } a(x) \cdot x \triangleright\{ \\
& \text { add }: x ?(n) \cdot x ?(m) \cdot x ![n+m], \\
& \ldots \\
& \operatorname{div}: x ?(n) \cdot x ?(m) \cdot x ![n \operatorname{div} m] \cdot x ![n \bmod m]\}
\end{aligned}
$$

whose typing of $x$ is just "longer" than $S_{\text {server } 1}$ :

$$
S_{\text {server } 2}=\&\langle\text { add : ?(int) ?(int)! [int] end, ..., div : ?(int) ?(int)! [int] ! [int] end }\rangle
$$

Can the concept of "being longer" be caught by means of subtyping? It is indeed tempting to try to extend the subtyping theory of session types introduced in [7] so that $S_{\text {client1 }}$ is a subtype of $S_{\text {client2 }}$, that we can assume for $x$ because of narrowing rule. However, as we shall argue in the sequel, this is not the case, and in fact the idea of extending a protocol represented by a type by some sort 
of subtyping in depth (whereas the subtyping of [7] is essentially in width w.r.t. branching/selection types) reveals to be of a different nature. Because of this we introduce a new relation among session types which we call prefix, axiomatize it and study its effect w.r.t. the "more liberal" system of session types in [20]. We choose the latter system as the basis of the present study also because it uses polarized channel names as private channels inside a session (like in [7] before). We profit of polarities to mark the server and the client end of a session, which we type differently w.r.t. the prefix relation.

The resulting system, when restricted to first-order sessions (i.e. transmitting just values and labels for selection among a branching of processes), satisfies a property we call weak compliance, which roughly says that a typed server cannot exhaust its actions on a channel before the dually typed client does. We call it weak compliance in contrast to the strong compliance considered in [4] because, as it is well known, session types do not guarantee deadlock freeness, so that we cannot expect that the client will also complete the interaction on its side. Technically the result is achieved by means of a simple semantics of session types interpreting types as sets of usages of channel names.

When considering higher order sessions (exchanging channel names of other sessions), the problem of variance of the types for input/output of a session is faced. We throughly study the possibility of adapting to the prefix relation the same variance rules that do hold in the case of subtyping in [7]. From the problems that arise we distill our solution, consisting in having both input and output types covariant w.r.t. the prefix, but we constrain the rules used to type the transmission of the (private) channel of a session. The resulting system nicely formalizes delegation, which in the world of object oriented programming is the act of transparently passing the interaction with a third party from one object providing a first part of a service, to another object that provides its continuation.

The full system of asymmetric session types retains all the basic properties of the original, symmetric system, namely subject reduction and error freeness. Unfortunately the semantics of first order types does not extend to higher order sessions, and we are able to prove a slightly weaker result of compliance in that case.

\section{The $\pi_{S}$-calculus and its Operational Semantics}

Session types are a type system for a dialect of the $\pi$-calculus introduced in [8]. From now on we will call $\pi_{S}$-calculus the $\pi$-calculus extended with primi-

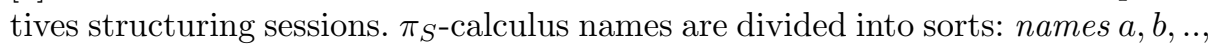
$x, y \ldots \in \mathcal{N},($ polarized $)$ channels $\kappa^{p}, \kappa^{\prime p}, \ldots \in \mathcal{C}$, labels $l, l^{\prime}, \ldots \in \mathcal{L}$. The term syntax is presented in Figure 1, where we omit definitions (and hence recursion) in order to focus on the essential issues and to keep the technical development simpler. Such a syntax is based on the second system introduced in [20], where polarized names are used for (private) channels, building over an idea that first appeared in [6] (a preliminary version of [7].) Notice that we take into account 


$\begin{array}{llll}e::= & \text { ground exprs } & p, q::=+\mid- & \text { polarities } \\ |x| \text { true } \mid \text { false }|0| 1 \mid \ldots & \text { vars and consts } & u, u^{\prime}::=x \mid \kappa & \\ |\operatorname{not}(e)| e+e^{\prime} \mid \ldots & \text { operators } & k, k^{\prime}::=a|x| \kappa \mid \kappa^{p} & \text { names, channels } \\ & & & \\ \pi::= & \text { prefixes } & P, Q::= & \text { processes } \\ \mid \text { request } a(x) & \text { session request } & \mid \mathbf{0} & \text { inaction } \\ \mid \text { accept } a(x) & \text { session acceptance } & \mid \pi \cdot P & \text { prefixed process } \\ \mid k ![e] & \text { data sending } & \mid k \triangleright\left\{l_{1}: P_{1}, . ., l_{n}: P_{n}\right\} & \text { label branching } \\ \mid k ?(x) & \text { data reception } & |P| Q & \text { parallel composition } \\ \mid \text { throw } k\left[k^{\prime}\right] & \text { channel sending } & \mid(\nu \kappa) P & \text { restriction } \\ \mid \text { catch } k(x) & \text { channel reception } & \mid \text { if } e \text { then } P \text { else } Q & \text { conditional branch } \\ \mid k \triangleleft l & \text { label selection } & & \end{array}$

Fig. 1. Term syntax of the $\pi_{S}$-calculus (without recursion).

the monadic version of the system, since our notions and results simply extend to the polyadic case.

Communications belonging to a session are opened through a public name and pursued through a private channel, specific to the session, which is created at connection time. The syntax used for the initiation of a session is:

$$
\text { request } a(x) \cdot P \mid \text { accept } a(x) \cdot Q
$$

A process opening a session by a request action over $a$ will be called a client w.r.t. the name $a$; symmetrically, a server w.r.t. $a$ opens the session using accept. Of course a process can be client and server w.r.t. different names, and even w.r.t. the same name that could be used to open several sessions.

A polarized channel name is a channel name decorated with a polarity: $\kappa^{p}$, $\kappa^{\bar{p}}$ where $p \in\{+,-\}$ and $\mp=-$ and $\bar{\mp}=+$. We use polarities not only, as in $[7$, $20]$, to couple the owners of a private channel, but also to distinguish between the client's and server's end of it ('-' for the client and '+' for the server). Indeed the process term in (1) reduces to:

$$
(\nu \kappa)\left(\left\{\kappa^{-} / x\right\} P \mid\left\{\kappa^{+} / x\right\} Q\right)
$$

where the channel name $\kappa$ is fresh. Note that occurrences of channels are always polarized (even in the scope of a binder). The only use of unpolarized channels is in the binder operator $(\nu \kappa)$, which binds all the channels $\kappa^{p}$ in its scope, regardless of $p$.

Free and bound names and channel names in $P$ are denoted, respectively, by $\operatorname{FN}(P)$ and $\operatorname{BN}(P)$ and defined as in [20]. Substitution and $\alpha$-congruence, written $P \equiv_{\alpha} Q$, are defined as usual.

The operational semantics of the calculus is usually defined in terms of a reduction relation up to structural congruence (see in particular [20], $\S 2.1$ and 
3.1). However, in order to make proofs simpler, we consider an equivalent LTS semantics. The labels of the LTS are from Act, the set of actions $\alpha$, defined by:

$$
\alpha::=a\left(\kappa^{+}\right)\left|a\left[\kappa^{-}\right]\right| \kappa^{p}(v)\left|\kappa^{p}[v]\right| \kappa^{p} \triangleleft l\left|\kappa^{p} \triangleright l\right| \kappa \nu k \mid \tau .
$$

where $v$ is ambiguous for $c, \kappa^{q}, \kappa$, and $k$ is either a name or a channel, possibly polarized; $\tau$ represents an internal action as for CCS. The dual action $\bar{\alpha}$ is defined only in the following cases:

$$
\overline{\kappa^{p}(v)}=\kappa^{\bar{p}}[v] \quad \overline{\kappa^{p}[v]}=\kappa^{\bar{p}}(v) \quad \overline{\kappa^{p} \triangleleft l}=\kappa^{\bar{p}} \triangleright l \quad \overline{\kappa^{p} \triangleright l}=\kappa^{\bar{p}} \triangleleft l
$$

The relation $P \stackrel{\alpha}{\longrightarrow} Q$ is then defined in Figure 2. It is an adaptation of the early semantics of the $\pi$-calculus (see e.g. [16]), where the symmetric of E-PAR, E-Close and E-Link are omitted.

$$
\begin{aligned}
& \frac{\kappa^{+}, \kappa^{-} \notin \mathrm{FN}(P)}{\text { accept } a(x) P \stackrel{a\left(\kappa^{+}\right)}{\longrightarrow}\left\{\kappa^{+} / x\right\} P} \text { E-ACC } \\
& \frac{\kappa^{+}, \kappa^{-} \notin \mathrm{FN}(P)}{\text { request } a(x) P \stackrel{a\left[\kappa^{-}\right]}{\longrightarrow}\left\{\kappa^{-} / x\right\} P} \text { E-REQ }
\end{aligned}
$$

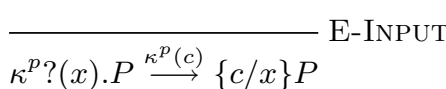

$$
\begin{aligned}
& \frac{l_{i} \in\left\{l_{1}, \ldots, l_{n}\right\}}{\kappa^{p} \triangleright\left\{l_{1}: P_{1}, . ., l_{n}: P_{n}\right\} \stackrel{\kappa^{p} \triangleright l_{i}}{\longrightarrow} P_{i}} \text { E-BRN } \\
& \frac{P \stackrel{\alpha}{\longrightarrow} P^{\prime} \quad \kappa \notin \alpha}{(\nu \kappa) P \stackrel{\alpha}{\longrightarrow}(\nu \kappa) P^{\prime}} \text { E-RES } \\
& \frac{P \stackrel{\alpha}{\longrightarrow} P^{\prime} \quad \operatorname{BN}(\alpha) \cap \mathrm{FN}(Q)=\emptyset}{P\left|Q \stackrel{\alpha}{\longrightarrow} P^{\prime}\right| Q} \text { E-PAR } \\
& \frac{e \downarrow \text { true }}{\text { if } e \text { then } P \text { else } Q \stackrel{\tau}{\longrightarrow} P} \text { E-IFT } \\
& \frac{P \stackrel{a\left(\kappa^{+}\right)}{\longrightarrow} P^{\prime} \quad Q \stackrel{a\left[\kappa^{-}\right]}{\longrightarrow} Q^{\prime}}{P \mid Q \stackrel{\tau}{\longrightarrow}(\nu \kappa)(P \mid Q)} \text { E-LiNK }
\end{aligned}
$$

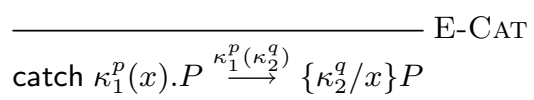

$$
\begin{aligned}
& \begin{array}{c}
\text { request } a(x) P \stackrel{a\left[\kappa^{-}\right]}{\longrightarrow}\left\{\kappa^{-} / x\right\} P \\
\frac{e \downarrow c}{\kappa^{p} ![e] \cdot P \stackrel{\kappa^{p}[c]}{\longrightarrow} P} \text { E-OUTPUT }
\end{array} \\
& \overline{\kappa^{p} \triangleleft l . P \stackrel{\kappa^{p} \triangleleft l}{\longrightarrow} P} \text { E-SEL } \\
& \frac{P \stackrel{\kappa_{1}^{p}\left[\kappa_{2}^{q}\right]}{\longrightarrow} P^{\prime} \quad \kappa_{1} \neq \kappa_{2}}{\left(\nu \kappa_{2}^{q}\right) P \stackrel{\kappa_{1}^{p} \nu \kappa_{2}^{q}}{\longrightarrow} P^{\prime}} \text { E-OPEN } \\
& \frac{P \stackrel{\kappa^{p}\left(\kappa^{\prime}\right)}{\longrightarrow} P^{\prime} \quad Q \stackrel{\kappa^{\bar{p}} \nu \kappa^{\prime}}{\longrightarrow} Q^{\prime}}{P \mid Q \stackrel{\tau}{\longrightarrow}\left(\nu \kappa^{\prime}\right)\left(P^{\prime} \mid Q^{\prime}\right)} \text { E-CLOSE } \\
& \frac{e \downarrow \text { false }}{\text { if } e \text { then } P \text { else } Q \stackrel{\tau}{\longrightarrow} Q} \text { E-IFF } \\
& \frac{P \stackrel{\alpha}{\longrightarrow} P^{\prime} \quad Q \stackrel{\bar{\alpha}}{\longrightarrow} Q^{\prime}}{P\left|Q \stackrel{\tau}{\longrightarrow} P^{\prime}\right| Q^{\prime}} \text { E-CoM }
\end{aligned}
$$

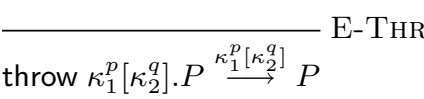

Fig. 2. Early LTS-operational semantics of $\pi_{S}$. 


$$
\begin{aligned}
& \bar{S}=S \text { if } S=\text { end } \quad \overline{\uparrow[S]}=\uparrow[S] \quad \overline{?(T) S}=![T] \bar{S} \quad \overline{![T] S}=?(T) \bar{S} \\
& \overline{\&\left\langle l_{1}: S_{1}, . ., l_{n}: S_{n}\right\rangle}=\oplus\left\langle l_{1}: \overline{S_{1}}, . ., l_{n}: \overline{S_{n}}\right\rangle \quad \overline{\oplus\left\langle l_{1}: S_{1}, . ., l_{n}: S_{n}\right\rangle}=\&\left\langle l_{1}: \overline{S_{1}}, \ldots, l_{n}: \overline{S_{n}}\right\rangle
\end{aligned}
$$

Fig. 3. Dual session types

By denoting with $P \longrightarrow Q$ the reduction semantics, as defined in [20]§3.1, the proof of the following can be provided by essentially mimicking that of the Harmony Lemma in [19].

Proposition 1. The LTS semantics and the reduction semantics are equivalent:

1. if $P \equiv \stackrel{\alpha}{\longrightarrow} P^{\prime}$ then $P \stackrel{\alpha}{\longrightarrow} \equiv P^{\prime}$;

2. $P \longrightarrow P^{\prime}$ if and only if $P \stackrel{\tau}{\longrightarrow} \equiv P^{\prime}$.

\section{Asymmetric Session Types}

Definition 1 (Types). The sets of types $\mathcal{T}$, and of session types $\mathcal{S} \mathcal{T}$, are defined according to the following grammar

$$
\begin{array}{rc}
\text { Type } & T::=\text { bool } \mid \text { nat } \mid \text { int } \mid \text { real }|S| \uparrow[S] \\
\text { Session type } & S::=?(T) S|![T] S| \&\left\langle l_{1}: S_{1}, \ldots, l_{n}: S_{n}\right\rangle \\
& \left|\oplus\left\langle l_{1}: S_{1}, \ldots, l_{n}: S_{n}\right\rangle\right| \text { end }
\end{array}
$$

A session type is first order if neither ?(T)S nor ![T]S occurs in it for any $T \in \mathcal{S T}$; it is higher order otherwise. We recall that the ordering of labels in $\&\left\langle l_{1}: S_{1}, \ldots, l_{n}: S_{n}\right\rangle$ and $\oplus\left\langle l_{1}: S_{1}, \ldots, l_{n}: S_{n}\right\rangle$ is immaterial. The operation $\bar{S}$ over $\mathcal{S T}$ is defined as in Figure 3. It is easy to check that $\overline{\bar{S}}=S$ for any $S \in \mathcal{S T}$.

Types are assigned to names according to the rules in Figure 4. Judgments have either the form $\Gamma \vdash x: T, \Gamma \vdash e: T$ or $\Gamma \vdash P \triangleright \Delta$, where $x \in \mathcal{N}, e$ is an expression, $P$ is a process and where $\Gamma$ and $\Delta$ are typing contexts, i.e. finite mappings from names to types. In particular, $\Gamma(x)=T$ where $\operatorname{dom}(\Gamma) \subseteq \mathcal{N}$, and $\Delta\left(\kappa^{p}\right)=S$ where $\operatorname{dom}(\Delta) \subseteq \mathcal{C}$. As in [8], $\Delta$ is called a typing. Note that $\kappa^{+}$and $\kappa^{-}$are considered as different, so that both may be in $\operatorname{dom}(\Delta)$ for some typing $\Delta$. Differently from [20]§3, the domain of a typing contains only polarized channels. A typing $\Delta$ is completed if $\Delta\left(\kappa^{p}\right)=$ end, for all $\kappa^{p} \in \operatorname{dom}(\Delta)$; balanced if $\Delta\left(\kappa^{p}\right)=\overline{\Delta\left(\kappa^{\bar{p}}\right)}$ whenever $\kappa^{p}, \kappa^{\bar{p}} \in \operatorname{dom}(\Delta)$; strictly balanced if it is balanced and $\kappa^{p} \in \operatorname{dom}(\Delta)$ implies $\kappa^{\bar{p}} \in \operatorname{dom}(\Delta)$. In case $\kappa^{p} \notin \operatorname{dom}(\Delta), \Delta \cdot \kappa^{p}: S$ is defined as the typing $\Delta^{\prime}$ such that $\Delta^{\prime}\left(\kappa^{p}\right)=S$ and $\Delta^{\prime}\left(\kappa^{\prime q}\right)=\Delta\left(\kappa^{\prime q}\right)$ if $\kappa^{\prime q} \neq \kappa^{p}$. $\Delta \cdot \Delta^{\prime}$ denotes the component wise extension of the dot operation, which is hence defined only if $\operatorname{dom}(\Delta) \cap \operatorname{dom}\left(\Delta^{\prime}\right)=\emptyset$. 


$$
\begin{aligned}
& \frac{\Gamma(x)=T}{\Gamma \vdash x: T} \text { T-NAmE } \frac{\Delta \text { completed }}{\Gamma \vdash \mathbf{0} \triangleright \Delta} \text { T-InACT } \quad \frac{\Gamma \vdash P \triangleright \Delta \quad \Gamma \vdash Q \triangleright \Delta^{\prime}}{\Gamma \vdash P \mid Q \triangleright \Delta \cdot \Delta^{\prime}} \text { T-PAR } \\
& \frac{\Gamma \vdash a: \uparrow[S] \quad \Gamma \vdash\left\{\kappa^{+} / x\right\} P \triangleright \Delta \cdot \kappa^{+}: S}{\Gamma \vdash \operatorname{accept} a(x) . P \triangleright \Delta} \mathrm{T}-\mathrm{ACC} \\
& \frac{\Gamma \vdash a: \uparrow[S] \quad \Gamma \vdash\left\{\kappa^{-} / x\right\} P \triangleright \Delta \cdot \kappa^{-}: \bar{S}}{\Gamma \vdash \text { request } a(x) . P \triangleright \Delta} \mathrm{T}-\mathrm{REQ} \\
& \frac{\Gamma \vdash P \triangleright \Delta \cdot \kappa^{p}: S \quad \Gamma \vdash e: T}{\Gamma \vdash \kappa^{p} ![e] . P \triangleright \Delta \cdot \kappa^{p}: ![T] S} \text { T-SEND } \frac{\Gamma \cdot x: T \vdash P \triangleright \Delta \cdot \kappa^{p}: S}{\Gamma \vdash \kappa^{p} ?(x) . P \triangleright \Delta \cdot \kappa^{p}: ?(T) S} \text { T-RCV } \\
& \frac{\left\{\Gamma \vdash P_{i} \triangleright \Delta \cdot \kappa^{p}: S_{i}\right\}_{i=1, \ldots, n}}{\Gamma \vdash \kappa^{p} \triangleright\left\{l_{1}: P_{1}, \ldots, l_{n}: P_{n}\right\} \triangleright \Delta \cdot \kappa^{p}: \&\left\langle l_{1}: S_{1}, \ldots, l_{n}: S_{n}\right\rangle} \text { T-BR } \\
& \frac{\Gamma \vdash P \triangleright \Delta \cdot \kappa^{p}: S_{i} \quad i \in\{1, \ldots n\}}{\Gamma \vdash \kappa^{p} \triangleleft l_{i} . P \triangleright \Delta \cdot \kappa^{p}: \oplus\left\langle l_{1}: S_{1}, \ldots, l_{n}: S_{n}\right\rangle} \text { T-SEL } \\
& \frac{\Gamma \vdash P \triangleright \Delta \cdot \kappa^{+}: S \cdot \kappa^{-}: \bar{S}}{\Gamma \vdash(\nu \kappa) P \triangleright \Delta} \mathrm{T}-\mathrm{NewS} \frac{\Gamma \vdash P \triangleright \Delta \kappa \notin \operatorname{dom}(\Delta)}{\Gamma \vdash(\nu \kappa) P \triangleright \Delta} \mathrm{T}_{-N e w S^{\prime}}
\end{aligned}
$$

Fig. 4. The First-Order Type System

Beside the restriction of typings $\Delta$ to polarized channel names, the main difference w.r.t. the system in $\S 3$ of [20] concerns rules T-ACC and T-REQ. In our system the bound name $x$ in the conclusion is substituted for the polarized channel name $\kappa^{p}$ appearing in the process $\left\{\kappa^{p} / x\right\} P$ of the premises. The polarity $p$ is ' + ' in T-ACC and ' - ' in T-REQ. Such requirements on polarities force to use the channel represented by $x$ as a server's or client's end of a session, respectively.

Session types enforce a perfect symmetry of the server and client actions via rules T-AcC, T-REQ and T-NewS: the first two, together with the additivity of rule T-PAR w.r.t. the left hand typing $\Gamma$, ensure that a connection opened through a name $a$ such that $a: \uparrow[S] \in \Gamma$ will use a channel $\kappa^{+}$of type $S$ on the server side, and a channel $\kappa^{-}$of type $\bar{S}$ on the client side. Since this channel is private and a process like (1) reduces to one like (2), the same correspondence is required to introduce the restriction $(\nu \kappa)$ in rule T-NEwS.

We wish now to formalize the bias toward the client by breaking such a symmetry, allowing sessions in which the client might do less than the server actually offers. A first attempt could consist in using subtyping (we write $T<$ : $T^{\prime}$ ), as introduced in [18] for I/O types and studied in [7] specifically for session types. 
For the sake of the subsequent discussion we recall that, w.r.t. the subtyping of I/O types, of which it saves the covariance of the input and contravariance of the output types, the theory in [7] includes:

$$
\frac{S_{i}<: S_{i}^{\prime}(\forall i \leq n) \quad n \leq m}{\&\left\langle l_{1}: S_{1}, . ., l_{n}: S_{n}\right\rangle<: \&\left\langle l_{1}: S_{1}^{\prime}, . ., l_{n}: S_{m}^{\prime}\right\rangle} \quad \frac{S_{i}<: S_{i}^{\prime} \quad(\forall i \leq n) \quad n \leq m}{\oplus\left\langle l_{1}: S_{1}, . ., l_{n}: S_{m}\right\rangle<: \oplus\left\langle l_{1}: S_{1}^{\prime}, . ., l_{n}: S_{n}^{\prime}\right\rangle}
$$

making the \& covariant and the $\oplus$ contravariant in width respectively (though both are covariant in depth).

Since we wish to embody the idea that a server might be ready to do more than it is declared by its "interface" $S$, we should add the axiom end $<: S$ to the theory in [7] and consider a restriction of the narrowing rule (the dual of the subsumption rule customary in the typed $\lambda$-calculus: see e.g. [17]) to positive channel names only:

$$
\frac{\Gamma \vdash P \triangleright \Delta \cdot \kappa^{+}: S \quad S^{\prime}<: S}{\Gamma \vdash P \triangleright \Delta \cdot \kappa^{+}: S^{\prime}} \text { T-Narrow }
$$

Now, being \& the dual of $\oplus$ and being ?(_)_ the dual of ![_] _ (with ?(_)_ covariant and ![_] then $\overline{S^{\prime}}<: \bar{S}$. However, this key property of subtyping relative to duality is incompatible with the axiom end $<: S$.

Theorem 1. There is no consistent theory of subtyping, extending the theory in [7], which includes the axiom end $<: S$ and satisfies the principle that if $S<: S^{\prime}$ then $\overline{S^{\prime}}<: \bar{S}$.

Proof. Toward a contradiction assume that if $S<$ : $S^{\prime}$ then $\overline{S^{\prime}}<$ : $\bar{S}$ holds; since end $<: \bar{S}$ is an instance of the axiom end $<: S$, for any $S$ we have that $S=\overline{\bar{S}}<$ : $\overline{\text { end }}=$ end. Therefore, by transitivity of $<$ :, it is $S<$ : $S^{\prime}$ for any $S, S^{\prime} \in \mathcal{S} \mathcal{T}$.

Theorem 1 leads us to introduce a new relation among session types: $S \prec S^{\prime}$, that we call prefix relation. Roughly, if $S \prec S^{\prime}$ then any interaction pattern typed by $S$ is the initial part of a pattern typed by $S^{\prime}$.

Definition 2 (Prefix Relation over First Order Session Types). The prefix relation over first-order session types, $S \prec S^{\prime}$ (read " $S$ is a prefix of $S^{\prime}$ ") is defined as the least preorder satisfying the following axiom and rules

$$
\begin{aligned}
& \overline{\text { end } \prec S} \quad \frac{S \prec S^{\prime}}{?(T) S \prec ?(T) S^{\prime}} \quad \frac{S \prec S^{\prime}}{![T] S \prec ![T] S^{\prime}} \\
& \frac{S_{i} \prec S_{i}^{\prime}(\forall i \leq n)}{\&\left\langle l_{1}: S_{1}, . ., l_{n}: S_{n}\right\rangle \prec \&\left\langle l_{1}: S_{1}^{\prime}, . ., l_{n}: S_{n}^{\prime}\right\rangle} \quad \frac{S_{i} \prec S_{i}^{\prime}(\forall i \leq n)}{\oplus\left\langle l_{1}: S_{1}, . ., l_{n}: S_{n}\right\rangle \prec \oplus\left\langle l_{1}: S_{1}^{\prime}, . ., l_{n}: S_{n}^{\prime}\right\rangle}
\end{aligned}
$$

To the system of Figure 4 we add the following rules:

$$
\frac{\Gamma \vdash P \triangleright \Delta \cdot \kappa^{+}: S \quad S^{\prime} \prec S}{\Gamma \vdash P \triangleright \Delta \cdot \kappa^{+}: S^{\prime}} \text { T-PREFS } \frac{\Gamma \vdash P \triangleright \Delta \cdot \kappa^{-}: S \quad S \prec S^{\prime}}{\Gamma \vdash P \triangleright \Delta \cdot \kappa^{-}: S^{\prime}} \text { T-PREFC }
$$


We remark that, while rule T-PREFS would be sound with $<$ : substituted for $\prec$ (it is just narrowing), T-PREFC is not. In fact the soundness of T-PREFC strictly depends on the fact that, differently than in the case of subtyping, \& and $\oplus$ are invariant in width, while they are both covariant in depth. This is clearly connected to the following property of $\prec$ which is the reason why Theorem 1 does not apply in the case of prefix.

Proposition 2. For any $S, S^{\prime} \in \mathcal{S} \mathcal{T}$, if $S \prec S^{\prime}$ then $\bar{S} \prec \overline{S^{\prime}}$.

We end the present section by establishing the basic correctness theorem for the first-order typing system. The definition of $P \equiv Q$ is the obvious extension of that of the $\pi$-calculus and can be found in [20].

Theorem 2 (Subject Reduction of the First-Order Typing System). If $\Gamma \vdash P \triangleright \Delta$ for a (strictly) balanced $\Delta$ and $P \stackrel{\tau}{\longrightarrow} \equiv P^{\prime}$ then $\Gamma \vdash P^{\prime} \triangleright \Delta^{\prime}$ for some (strictly) balanced $\Delta^{\prime}$.

\section{Compliance up to Deadlock}

According to [4] a client is "strongly compliant" with a service whenever it completes all direct interaction sessions with the service. On the other hand, as remarked in [7], session types do not enforce deadlock freeness in general: a client might be not strongly compliant because a deadlock occurs that prevents the session to proceed properly. As a matter of fact more is needed to guarantee deadlock freeness $[12,13]$ or even the weaker progress property [5]. Since we work essentially with the original system, we can only expect a weaker concept of compliance to be warranted for typable systems, up to deadlock occurrences:

Weak Compliance Property: a server cannot exhaust its actions on a channel before the corresponding client does.

To state and prove a result about weak compliance for typable systems we use some machinery to extract from a process term $P$ the intended usage of a channel name $\kappa^{p}$ in $P$, forgetting about anything else which could incidentally cause a block and prevent the full exploitation of the capabilities of $P$ using $\kappa^{p}$. This is inspired to the idea of using parallel free CCS terms to describe the usage of a channel in $[9,12]$, and to the theory of contracts [4], where CCS terms describe the protocol part of a contract.

Definition 3 (Usages). Usages are defined by the grammar:

$$
U, V::=0|\ell . U| U+V
$$

where $\ell$ is either input, output or $l, \bar{l}$ for some label $l$; we also assume input $=$ output and $\overline{\text { output }}=$ input. Call Usg the set of usages; over Usg we define an LTS by the rules:

$$
\stackrel{U \stackrel{\ell}{\longmapsto} U^{\prime}}{\ell . U \stackrel{\ell}{\longmapsto} U} \quad \frac{\ell}{U+V \stackrel{\ell}{\longmapsto} U^{\prime}}
$$


Over Usg it is defined a binary relation $\prec$ :

$$
U \prec V \quad \Leftrightarrow \quad\left[U \stackrel{\ell}{\longmapsto} U^{\prime} \Longrightarrow V \stackrel{\ell}{\longmapsto} V^{\prime}\right] .
$$

We overload the notation $\prec$ by speaking of prefix relation among usages as well as among types. Notice that we do not need to define $\prec$ on Usg as a full simulation, since for our result we need only to check about the outermost action having as subject a specified channel name.

Usages and types are connected in the sense that the type of a server end $\kappa^{+}$is a lower bound to its usage, while that of a client end $\kappa^{-}$is an upper bound, which is the contents of Theorem 3 below. To prove that we interpret session types as sets of usages, and connect the usage $U$ to the capabilities of $P$ restricted to the channel $\kappa^{p}$ via the notion of trace.

Definition 4 (Semantics of First-Order Session Types). Let $S \in \mathcal{S} \mathcal{T}$; then $\llbracket S \rrbracket$ is the set of usages defined as follows:

$$
\begin{aligned}
& \llbracket \text { end } \rrbracket=\{0\} ; \quad \llbracket ?(T) S \rrbracket=\{\text { input. } U \mid U \in \llbracket S \rrbracket\} ; \quad \llbracket ![T] S \rrbracket=\{\text { output. } U \mid U \in \llbracket S \rrbracket\} \\
& \llbracket \&\left\langle l_{1}: S_{1}, \ldots, l_{n}: S_{n}\right\rangle \rrbracket=\left\{\sum_{i \in I} l_{i} . U_{i} \mid\{1, \ldots, n\} \subseteq I \wedge \forall i \in\{1, \ldots, n\} . U_{i} \in \llbracket S_{i} \rrbracket\right\} \\
& \llbracket \oplus\left\langle l_{1}: S_{1}, \ldots, l_{n}: S_{n}\right\rangle \rrbracket=\left\{\bar{l} . U \mid \exists i \in\{1, \ldots, n\} . l=l_{i} \wedge U \in \llbracket S_{i} \rrbracket\right\} .
\end{aligned}
$$

By Proposition 1 , we can safely abbreviate $P \stackrel{\tau}{\longrightarrow} Q$ by $P \longrightarrow Q$. In the following $\Longrightarrow$ denotes the reflexive and transitive closure of $\longrightarrow$ and $\stackrel{\alpha}{\Longrightarrow}$ denotes the composition $\Longrightarrow \stackrel{\alpha}{\Longrightarrow} \Longrightarrow$. Let $\psi=\alpha_{1} \cdots \alpha_{n}$ be in Act $^{*}$ ( $\epsilon$ will denote the empty sequence), then $P \stackrel{\psi}{\Longrightarrow} Q$ abbreviates $P \stackrel{\alpha_{1}}{\Longrightarrow} \cdots \stackrel{\alpha_{n}}{\Longrightarrow} Q$.

Definition 5 (Traces). The set $\operatorname{Tr}(P)$ of traces of $P$ is defined as

$$
\operatorname{Tr}(P)=\left\{\psi \in \mathbf{A c t}^{*} \mid \exists Q . P \stackrel{\psi}{\Longrightarrow} Q\right\} .
$$

If $\psi \in \operatorname{Tr}(P)$ then let $\psi\left\lceil\kappa^{p}\right.$ be the string of actions $\alpha$ in $\psi$ whose subject is $\kappa^{p}$, and write $\operatorname{Tr}\left(P, \kappa^{p}\right)=\left\{\psi\left|\kappa^{p}\right| \psi \in \operatorname{Tr}(P)\right\}$.

The mapping usg : Act $\rightarrow$ Usg is defined by:

$$
\begin{gathered}
\operatorname{usg}\left(\kappa^{p} \triangleleft l\right)=\bar{l} ; \quad \operatorname{usg}\left(\kappa^{p} \triangleright l\right)=l ; \quad \operatorname{usg}\left(\kappa^{p}(v)\right)=\text { input } \\
\operatorname{usg}\left(\kappa^{p}[v]\right)=\operatorname{usg}\left(\kappa^{p} \nu \kappa^{\prime q}\right)=\text { output }
\end{gathered}
$$

This map extends pointwise to Act* by imposing usg $(\epsilon)=0$. We shall write $\operatorname{simply} \operatorname{usg}(\psi)$ for $\psi \in$ Act* $^{*}$.

Lemma 1. Let $\phi \in$ Act $^{*}$, then $\overline{\operatorname{usg}(\phi)}=\operatorname{usg}(\bar{\phi})$.

Let $\mathcal{A}, \mathcal{B} \subseteq \mathbf{U s g}$, then define:

$$
\begin{aligned}
& \mathcal{A} \sqsubseteq * \mathcal{B} \Leftrightarrow \exists U \in \mathcal{A} .\left[U \stackrel{\ell}{\longmapsto} U^{\prime} \Longrightarrow \exists V \in \mathcal{B} . V \stackrel{\ell}{\longmapsto} V\right] \\
& \mathcal{A} \sqsubseteq^{*} \mathcal{B} \Leftrightarrow \exists V \in \mathcal{B} \forall U \in \mathcal{A} . U \prec V .
\end{aligned}
$$


Theorem 3 (Soundness of First Order Type Interpretation). Let $\Theta ; \Gamma \vdash$ $P \triangleright \Delta$ be derivable, then:

1. if $\kappa^{+} \in \operatorname{dom}(\Delta)$ and $\mathcal{A}=\left\{\operatorname{usg}(\psi) \mid \psi \in \operatorname{Tr}\left(P, \kappa^{+}\right)\right\}$then $\llbracket \Delta\left(\kappa^{+}\right) \rrbracket \sqsubseteq_{*} \mathcal{A}$;

2. if $\kappa^{-} \in \operatorname{dom}(\Delta)$ and $\mathcal{B}=\left\{\operatorname{usg}(\psi) \mid \psi \in \operatorname{Tr}\left(P, \kappa^{-}\right)\right\}$then $\mathcal{B} \sqsubseteq^{*} \llbracket \Delta\left(\kappa^{-}\right) \rrbracket$.

Corollary 1 (Weak Compliance). Let $\Gamma \vdash P \triangleright \Delta$ be derivable for some strictly balanced $\Delta$. If $P \stackrel{\alpha}{\Longrightarrow} P^{\prime}$ for some $P^{\prime}$ and the subject of $\alpha$ is some $\kappa^{-}$then $\alpha^{\prime} \psi^{\prime} \in \operatorname{Tr}\left(P, \kappa^{+}\right)$, for some $\psi^{\prime}$ and $\alpha^{\prime}$ such that usg $\left(\alpha^{\prime}\right)=\operatorname{usg}(\bar{\alpha})$.

Corollary 1 does not extend straightforwardly to higher order sessions and types: this is due to the fact that the object of a throw action is associated in the typing $\Delta$ to some session type that does not correspond to any usage of the channel in that term.

\section{Delegation via Higher-Order Sessions}

Mobility in the $\pi_{S}$-calculus is formalized by the primitives throw and catch, which, respectively, send and receive channel names. According to [8], these primitives enable to implement delegation, that is the ability for a process to pass a session to some third party which is in charge of continuing the interaction. Such a behaviour is reflected in the typing of the subjects of throw and catch by, respectively, ! $\left[S^{\prime}\right] S$ and ? $\left(S^{\prime}\right) S$. Adapting the rules in $[8,20]$ to our system, where a polarized channel name $\kappa_{2}^{q}$ is replaced by an unpolarized $x$ in the body of the binding catch $\kappa^{p}(x) . P$, we obtain:

$$
\begin{gathered}
\frac{\Gamma \vdash P \triangleright \Delta \cdot \kappa_{1}^{p}: S \quad S^{\prime} \neq \text { end }}{\Gamma \vdash \text { throw } \kappa_{1}^{p}\left[\kappa_{2}^{q}\right] \cdot P \triangleright \Delta \cdot \kappa_{1}^{p}: !\left[S^{\prime}\right] S \cdot \kappa_{2}^{q}: S^{\prime}} \mathrm{T}^{-\mathrm{THR}^{\prime}} \\
\frac{\Gamma \vdash\left\{\kappa_{2}^{q} / x\right\} P \triangleright \Delta \cdot \kappa_{1}^{p}: S \cdot \kappa_{2}^{q}: S^{\prime}}{\Gamma \vdash \operatorname{catch} \kappa_{1}^{p}(x) \cdot P \triangleright \Delta \cdot \kappa_{1}^{p}: ?\left(S^{\prime}\right) S} \mathrm{~T}^{-\mathrm{CAT}^{\prime}}
\end{gathered}
$$

where in rule T-CAT ${ }^{\prime}$ the implicit assumption that $\Delta \cdot \kappa_{1}^{p}: !\left[S^{\prime}\right] S \cdot \kappa_{2}^{q}: S^{\prime}$ is well formed implies that $\kappa^{q} \notin \mathrm{FN}(P)$.

In presence of T-PREFC, however, the above typing rules force! $\left[S^{\prime}\right] S$ and $?\left(S^{\prime}\right) S$ to behave invariantly in $S^{\prime}$ w.r.t. prefix relation. In fact in rule T-CAT ${ }^{\prime}$ the information about the polarity of $\kappa_{2}^{q}$, while needed in the typing of $\left\{\kappa_{2}^{q} / x\right\} P$, is present in the conclusion only as input type for $\kappa_{1}^{p}$. As a consequence, any process willing to send $\kappa_{2}^{q}$ via $\kappa_{1}^{\bar{p}}$ to catch $\kappa_{1}^{p}(x) . P$ cannot make any assumption about the actual usage of $\kappa_{2}^{q}$ in $\left\{\kappa_{2}^{q} / x\right\} P$, either as a client or as a server end, and therefore the session type $S^{\prime}$ can be neither shorter (as in the case $q=+$ ) nor larger (when $q=-$ ) than the actual usage of $\kappa_{2}^{q}$.

Since invariance would be unreasonably restrictive, we need to establish a relation between the $p$ in the conclusion and the $q$ in the premise of rule T-CAT'. As explanatory example, let $P^{\prime}=_{\text {def }}\left\{\kappa_{2}^{q} / x\right\} P$ and consider:

$$
R={ }_{\text {def }} Q \mid \text { throw } \kappa_{1}^{\bar{p}}\left[\kappa_{2}^{q}\right] \mid \text { catch } \kappa_{1}^{p}(x) P
$$


where $\kappa_{2}^{\bar{q}} \in \mathrm{FN}(Q)$.

Suppose that, w.r.t. the prefix relation, ! $\left[S^{\prime}\right] S$ is contravariant in $S^{\prime}$ and covariant in $S$, and that ? $\left(S^{\prime}\right) S$ is covariant both in $S^{\prime}$ and in $S$ (this is actually the case w.r.t. subtyping I/O types in [18], and session types in [7].) Then we study under what conditions $\left(\nu \kappa_{1}\right)\left(\nu \kappa_{2}\right) R$ is safely typable, in the sense that both Weak Compliance and Error-Freeness (see [8] and Theorem 4 below) are preserved.

Let $\Gamma \vdash$ catch $\kappa_{1}^{p}(x) P \triangleright \Delta \cdot \kappa_{1}^{p}: ?\left(S_{1}\right)$ end, $\Gamma \vdash P^{\prime} \triangleright \Delta \cdot \kappa_{2}^{q}: S_{2}$ and $\Gamma \vdash$ $Q \triangleright \Delta^{\prime} \cdot \kappa_{2}^{\bar{q}}: \overline{S_{3}}$ be derivable, and suppose that $S_{1} \prec S_{2}$ and $S_{1} \prec S_{3}$, but that $S_{2}$ and $S_{3}$ are incompatible (for example, $S_{1}=$ ?(int)end, $S_{2}=$ ?(int)! [int]end and $S_{3}=$ ?(int)![bool] end).

Case $p=-, q=+$.

$$
\begin{aligned}
& \frac{\Gamma \vdash P^{\prime} \triangleright \Delta \cdot \kappa_{2}^{+}: S_{2} \quad S_{1} \prec S_{2}}{\Gamma \vdash P^{\prime} \triangleright \Delta \cdot \kappa_{2}^{+}: S_{1}} \text { T-PREFS } \\
& \frac{\Gamma \vdash \text { catch } \kappa_{1}^{-}(x) P \triangleright \Delta \cdot \kappa_{1}^{-}: ?\left(S_{1}\right) \text { end }}{\Gamma-C_{A T}^{\prime} \quad ?\left(S_{1}\right) \text { end } \prec ?\left(S_{3}\right) \text { end }} \text { T-PrefcC }
\end{aligned}
$$

Now, since $\Gamma \vdash$ throw $\kappa_{1}^{+}\left[\kappa_{2}^{+}\right] \triangleright \kappa_{1}^{+}: !\left[S_{3}\right]$ end $\cdot \kappa_{2}^{+}: S_{3}$ by T-THR', we have that $\Gamma \vdash R \triangleright \Delta \cdot \Delta^{\prime} \cdot \kappa_{1}^{-} ?\left(S_{3}\right)$ end $\cdot \kappa_{1}^{+} !\left[S_{3}\right]$ end $\cdot \kappa_{2}^{-}: \overline{S_{3}} \cdot \kappa_{2}^{+}: S_{3}$ is derivable so that $\left(\nu \kappa_{1}\right)\left(\nu \kappa_{2}\right) R$ typechecks, but it is unsafe, as $Q$ might require an interaction over $\kappa_{2}^{-}$which is exactly typed by $\overline{S_{3}}$ while $P^{\prime}$, that will receive $\kappa_{2}^{+}$, is ready to respect just the unrelated protocol $S_{2}$.

Case $p=-, q=-$ : using T-PrefC with the premise $S_{1} \prec S_{3}$ and T-PrefS with the premise ! $\left[S_{2}\right]$ end $\prec !\left[S_{1}\right]$ end (implied by $S_{1} \prec S_{2}$ and the contravariance of ![ ]) we have:

$$
\frac{\Gamma \vdash \text { throw } \kappa_{1}^{+}\left[\kappa_{2}^{-}\right] \triangleright \kappa_{1}^{+}: !\left[S_{1}\right] \text { end } \cdot \kappa_{2}^{-}: S_{1} \quad S_{1} \prec S_{3}}{\left.\frac{\Gamma \vdash \text { throw } \kappa_{1}^{+}\left[\kappa_{2}^{-}\right] \triangleright \kappa_{1}^{+}: !\left[S_{1}\right] \text { end } \cdot \kappa_{2}^{-}: S_{3}}{\Gamma \vdash \text { throw } \kappa_{1}^{+}\left[\kappa_{2}^{-}\right] \triangleright \kappa_{1}^{+}: !\left[S_{2}\right] \text { end } \cdot \kappa_{2}^{-}: S_{3}} \quad ! S_{2}\right] \text { end } \preccurlyeq !\left[S_{1}\right] \text { end }}
$$

But since $\Gamma \vdash$ catch $\kappa_{1}^{-}(x) P \triangleright \Delta \cdot \kappa_{1}^{-}: ?\left(S_{2}\right)$ end is derivable from $\Gamma \vdash P^{\prime} \triangleright \Delta \cdot \kappa_{2}^{-}$: $S_{2}$ by T-CAT ${ }^{\prime}$, we have that $\Gamma \vdash R \triangleright \Delta \cdot \Delta^{\prime} \cdot \kappa_{1}^{-}$? $\left(S_{2}\right)$ end $\cdot \kappa_{1}^{+} !\left[S_{2}\right]$ end $\cdot \kappa_{2}^{-}: \overline{S_{3}} \cdot \kappa_{2}^{+}$: $S_{3}$ is derivable but unsafe, because the server in $Q$ will be unable to provide a service with protocol $\overline{S_{2}}$ as required by the client in $P^{\prime}$.

Case $p=+, q=-$.

$$
\frac{\frac{\Gamma \vdash P^{\prime} \triangleright \Delta \cdot \kappa_{2}^{-}: S_{2}}{\Gamma \vdash \operatorname{catch} \kappa_{1}^{+}(x) P \triangleright \kappa_{1}^{+}: ?\left(S_{2}\right) \text { end }} \quad ?\left(S_{1}\right) \text { end } \preccurlyeq ?\left(S_{2}\right) \text { end }}{\Gamma \vdash \operatorname{catch} \kappa_{1}^{+}(x) P \triangleright \kappa_{1}^{+}: ?\left(S_{1}\right) \text { end }} \text { T-PREFS }
$$

This time we derive $\Gamma \vdash$ throw $\kappa_{1}^{-}\left[\kappa_{2}^{-}\right] \triangleright !\left[S_{1}\right]$ end $\cdot \kappa_{2}^{-}: S_{3}$ from $\Gamma \vdash$ throw $\kappa_{1}^{-}\left[\kappa_{2}^{-}\right] \triangleright$ ! $\left[S_{1}\right]$ end $\cdot \kappa_{2}^{-}: S_{1}$ by $S_{1} \prec S_{3}$ and T-PrEFC; hence we have:

$$
\Gamma \vdash R \triangleright \Delta \cdot \Delta^{\prime} \cdot \kappa_{1}^{-} ?\left(S_{1}\right) \text { end } \cdot \kappa_{1}^{+} !\left[S_{1}\right] \text { end } \cdot \kappa_{2}^{-}: \overline{S_{3}} \cdot \kappa_{2}^{+}: S_{3}
$$




$$
\begin{gathered}
\frac{\Gamma \vdash\left\{\kappa_{2}^{p} / x\right\} P \triangleright \Delta \cdot \kappa_{1}^{p}: S \cdot \kappa_{2}^{p}: S^{\prime}}{\Gamma \vdash \operatorname{catch} \kappa_{1}^{p}(x) \cdot P \triangleright \Delta \cdot \kappa_{1}^{p}: ?\left(S^{\prime}\right) S} \mathrm{~T}^{-\mathrm{CAT}_{p}} \\
\frac{\Gamma \vdash P \triangleright \Delta \cdot \kappa_{1}^{\bar{p}}: S}{\Gamma \vdash \operatorname{throw} \kappa_{1}^{\bar{p}}\left[\kappa_{2}^{p}\right] \cdot P \triangleright \Delta \cdot \kappa_{1}^{\bar{p}}: !\left[S^{\prime}\right] S \cdot \kappa_{2}^{p}: S^{\prime}} \mathrm{T}^{-\mathrm{THR}_{p}}
\end{gathered}
$$

Fig. 5. The type rules for Higher-Order sessions

so that $\left(\nu \kappa_{1}\right)\left(\nu \kappa_{2}\right) R$ typechecks but it is unsafe since the server $\kappa_{2}^{+}$in $Q$ will provide a service of type $\overline{S_{3}}$ to the client $\kappa_{2}^{-}$in $P^{\prime}$, whose protocol is $S_{2}$.

Case $p=+, q=+$ : by the previous derivation (where the polarity of $\kappa_{2}$ does not play any role) we have $\Gamma \vdash$ catch $\kappa_{1}^{+}(x) P \triangleright \kappa_{1}^{+}: ?\left(S_{1}\right)$ end; on the other hand we have $\Gamma \vdash$ throw $\kappa_{1}^{-}\left[\kappa_{2}^{+}\right] \triangleright \kappa_{1}^{-}:$! [S $\left.S_{1}\right]$ end $\cdot \kappa_{2}^{+}: S_{3}$ from $\Gamma \vdash$ throw $\kappa_{1}^{-}\left[\kappa_{2}^{+}\right] \triangleright \kappa_{1}^{-}$: ! $\left[S_{3}\right]$ end $\cdot \kappa_{2}^{+}: S_{3}$ by T-PREFC using the contravariance of ! [ ], which implies that ! $\left[S_{3}\right]$ end $\prec$ ! $\left[S_{1}\right]$ end. Hence we obtain that $\Gamma \vdash R \triangleright \Delta \cdot \Delta^{\prime} \cdot \kappa_{1}^{-}$? $\left(S_{1}\right)$ end $\kappa_{1}^{+}$! [ $\left.S_{1}\right]$ end $\cdot \kappa_{2}^{-}: \overline{S_{3}} \cdot \kappa_{2}^{+}: S_{3}$ with a similar mismatch as before, but having the server in $P^{\prime}$ and the client in $Q$.

We observe that in the cases when $p \neq q$ problems arise because of an inner incoherence of the principle of delegation for those particular client/server asymmetric interactions; namely when the throw process is a client(server) which delegates an interaction with respect to which it is working as client(server). For the cases when $p=q$, instead, the problems depend only on the contravariance of the output type ![ ]. So, a way out is to assume covariance of both input and output higher-order session types (see Definition 6) and put the equality of polarities of the subject and the object of a catch action (and consequently the duality of them in case of a throw action) into the typing rules (see Figure 5).

Definition 6 (Prefix Relation over Higher Order Session Types). The prefix relation over $\mathcal{S} \mathcal{T}$ is obtained by extending Definition 2 by:

$$
\frac{S_{1}^{\prime} \prec S_{2}^{\prime} \quad S_{1} \prec S_{2}}{!\left[S_{1}^{\prime}\right] S_{1} \prec !\left[S_{2}^{\prime}\right] S_{2}} \quad \frac{S_{1}^{\prime} \prec S_{2}^{\prime} \quad S_{1} \prec S_{2}}{?\left(S_{1}^{\prime}\right) S_{1} \prec ?\left(S_{2}^{\prime}\right) S_{2}}
$$

In the rest of this section we report on results that prove the soundness of the proposed system. Session type system ensure error freeness. To define errors observe that any process term is structurally congruent to a term of the shape $(\nu \boldsymbol{\kappa})\left(P_{1}|\cdots| P_{n}\right)$, where the $P_{i}$ are prefixed processes (including branching) or selections of the shape if $b$ then $Q$ else $R$. The $P_{i}$ are said to be in head position. If $\kappa^{p}$ is the subject of the prefix of a process $P_{i}$ we say that $P_{i}$ is a $\kappa$-process. The parallel of dual $\kappa$-processes is a $\kappa$-redex.

Definition 7 (Error Freeness). A process $P$ is an error if there exists a channel $\kappa$ such that either two $\kappa$-processes which do not form a $\kappa$-redex occur in $P$ in head position, or there are more than two $k$-processes in head position. 
A process $P$ is error free if there exists no $Q$ such that $P \stackrel{*}{\longrightarrow} Q$ which is an error.

The following result, proved in [8] for the original system, also holds in the asymmetric case.

Theorem 4 (Error Freeness). If $\Gamma \vdash P \triangleright \Delta$ then $P$ is error free.

As said before, besides error freeness, one of the most relevant properties of asymmetric systems is the Weak Compliance Property. In presence of Higherorder, however, this property does not hold in its full sense, as in Corollary 1. A simple counterexample can help to understand where the problem lies:

$$
\text { accept } a(y) \text {.request } b(x) \text {.throw } x[y] \quad \mid \text { request } a(y) . y ?(n)
$$

where the process request $a(y) \cdot y ?(n)$ is a client just needing a value. The server to which such a client can connect to in order to get the needed value is accept $a(y)$.request $b(x)$.throw $x[y]$. Such a server accepts the connection request from the client and immediately try to delegate the production of the value for the client to another server (we can look at the initial process (4) as a simplified version of the system CalcClient | CalcServer 5 ).

It is not difficult to check that process (4) is typable in an empty session environment, using $\{b: \uparrow[?(![$ int $]$ ![bool $])], a: \uparrow[![$ int $]]\}$ as type environment. It reduces to the running process $(\nu \kappa)$ (request $b(x)$.throw $\left.x\left[\kappa^{+}\right] \quad \mid \kappa^{-} ?(n)\right)$ where we have a $\kappa^{-}$-process in head position, but in which we can get an actual dual $\kappa^{+}$process only in case a typable server is added to the system. Such a potentiality is represented by the presence in the system of the process throw $x\left[\kappa^{+}\right]$.

The notion of "potential" $\kappa^{+}$-process is expressed by the following definition.

Definition 8 (Potential $\kappa^{+}$-process generator).

$A$ potential $\kappa^{+}$-process generator is any process of the form throw $k\left[\kappa^{+}\right] . Q$

A process $\mathrm{P}$ is initial if does not contain any channel name $\kappa$ neither free nor bound. A process $\mathrm{P}$ is running if there exists an initial $\mathrm{Q}$ such that: $Q \stackrel{*}{\rightarrow} P$

Theorem 5 (Higher-order Weak Compliance). Let $P$ be a running process which is a derivative of some typed initial process. If $P$ contains a $\kappa^{-}$process in head position, then it includes either a dual $\kappa^{+}$-process (though not necessarily in head position) or a potential $\kappa^{+}$-process generator.

\section{Related Work and Conclusion}

The main sources of this work are [8,20] and [7] on session types, and [4] for the idea of formalizing the protocols of asymmetric client/server interaction. With respect to these works we do not establish stronger results, rather we address a similar issue in a more complex setting, where processes exchange values and channels themselves, allowing for mobility and delegation. 
A recent contribution which is close to our development, especially for the type interpretation, is [3]. The semantics proposed there is far more complex then ours, and different because of use of internal choice that, we think, does not model properly the label selection and its typing in the session type systems (for an attempt to establish a weak form of correspondence between contracts, that use internal choice as well, and session types see [14]).

Introducing the relation of prefix in the rules of the systems breaks the symmetry of session type systems studied so far, but surprisingly enough does not destroy the basic properties of the system, namely subject reduction and error freeness. Nonetheless a great obstacle remains, which is connected to the fact that ordinary session types do not guarantee deadlock-freeness in the sense of $[10,12]$.

Even in presence of these limitations, we think that the system illustrated in this paper deserves interest. In fact the processes that can be represented in the $\pi$-calculus with sessions are far richer than those considered in the theory of contracts. They also are interesting w.r.t. the Service Centered Calculus (SCC) [1] and of recent proposals to detect deadlock freedom in SCC via type systems, e.g. [2].

\section{References}

1. M. Boreale, R. Bruni, L. Caires, R. D. Nicola, I. Lanese, M. Loreti, F. Martins, U. Montanari, A. Ravara, D. Sangiorgi, V. Vasconcelos, and G. Zavattaro. SCC: a Service Centered Calculus. In WS-FM 2006, volume 4184 of $L N C S$, pages 38-57. Springer-Verlag, 2006.

2. R. Bruni and L. Mezzina. Types and Deadlock Freedom in a Calculus of Services, Sessions and Pipelines. In Proc. of AMAST 2008, LNCS, to appear.

3. G. Castagna, M. Dezani-Ciancaglini, E. Giachino, and L. Padovani. General Session Types. url: http://www.sti.uniurb.it/padovani/publications.html, 2008.

4. G. Castagna, N. Gesbert, and L. Padovani. A theory of contracts for web services. In POPL '08, 35th ACM Symposium on Principles of Programming Languages, jan 2008.

5. M. Dezani-Ciancaglini, U. de' Liguoro, and N. Yoshida. On Progress for Structured Communications. In G. Barthe and C. Fournet, editors, TGC'07, volume 4912 of $L N C S$, pages 257-275. Springer, 2008.

6. S. Gay and M. Hole. Types and Subtypes for Client-Server Interactions. In ESOP'99, volume 1576 of LNCS, pages 74-90. Springer-Verlag, 1999.

7. S. Gay and M. Hole. Subtyping for Session Types in the Pi-Calculus. Acta Informatica, 42(2/3):191-225, 2005.

8. K. Honda, V. T. Vasconcelos, and M. Kubo. Language Primitives and Type Disciplines for Structured Communication-based Programming. In ESOP'98, volume 1381 of LNCS, pages 22-138. Springer-Verlag, 1998.

9. A. Igarashi and N. Kobayashi. A Generic Type System for the Pi-Calculus. Theoretical Computer Science, 311(1-3):121-163, 2004.

10. N. Kobayashi. A Type System for Lock-Free Processes. Information and Computation, 177:122-159, 2002.

11. N. Kobayashi. Type Systems for Concurrent Programs. In Formal Methods at the Crossroads, volume 2757 of LNCS, pages 439-453. Springer-Verlag, 2003. 
12. N. Kobayashi. A New Type System for Deadlock-Free Processes. In CONCUR'06, volume 4137 of $L N C S$, pages 233-247. Springer-Verlag, 2006.

13. N. Kobayashi. Type Systems for Concurrent Programs. Extended version of [11], Tohoku University, 2007.

14. C. Laneve and L. Padovani. The pairing of contracts and session types. In Concurrency, Graphs and Models, volume 5065 of Lecture Notes in Computer Science, pages 681-700, 2008.

15. G. Meredith and S. Bjorg. Contracts and types. Commun. ACM, 46(10):41-47, 2003.

16. J. Parrow. An introduction to the $\pi$-calculus. In A. Ponse, S. Smolka, and J. Bergstra, editors, Handbook of Process Algebra, chapter 8, pages 479-544. Elsevier, 2001.

17. B. C. Pierce. Types and Programming Languages. MIT Press, 2002.

18. B. C. Pierce and D. Sangiorgi. Typing and subtyping for mobile processes. In Logic in Computer Science, 1993. Full version in Mathematical Structures in Computer Science, Vol. 6, No. 5, 1996.

19. D. Sangiorgi and D. Walker. The $\pi$-calculus. A Theory of Mobile Processes. CUP, 2001.

20. N. Yoshida and V. T. Vasconcelos. Language Primitives and Type Disciplines for Structured Communication-based Programming Revisited. In SecReT'06, volume 171 of ENTCS, pages 73-93. Elsevier, 2007. 